\title{
45. The Poincaré Lemma for a Variation of Polarized Hodge Structure
}

\author{
By Masaki KASHIWARA and Takahiro KAWAI \\ Research Institute for Mathematical Sciences, Kyoto University \\ (Communicated by Kôsaku YosidA, M. J. A., June 11, 1985)
}

o. The purpose of this note is to present the Poincaré lemma for a variation of Hodge structure. The result was first proved in a pioneering work of Zucker ([4]) in the one-dimensional case. Cattani and Kaplan [1] has recently announced a generalization in the case of dimension 2 and weight 1.

Our main result (Theorem 1) shows the coincidence of the intersection cohomology groups and the $L^{2}$-cohomology groups associated with a variation of Hodge structure in higher dimensional case, thus generalizing the Poincaré lemma due to Zucker to the higher dimensional case. Note, however, that the holomorphic Poincaré lemma in the sense of [4] does not hold in the higher dimensional case. (See [1].) The proof of Theorem 1 is based on an algebraic result (Theorem 2), which is announced by Cattani and Kaplan [1] in the two-dimensional case.

1. Let $X$ be an $n$-dimensional complex manifold, $Y$ a normally crossing hypersurface and $\left(H_{Z}, F, S\right)$ a variation of polarized Hodge structure of weight $w$ over $X \backslash Y$, that is, $H_{Z}$ is a local system on $X \backslash Y, S$ is a nondegenerate bilinear form on $H_{Q}$ and $F$ is a finite filtration of $\mathcal{O}_{X \backslash Y} \otimes H_{Z}$ by holomorphic vector bundles such that at any point $x$ in $X \backslash Y$ the stalk of $\left(H_{Z}, F, S\right)$ gives a polarized Hodge structure and $v F^{p} \subset F^{p-1}$ for any holomorphic vector field $v$ and any $p$. Then $H$ gives a $C^{\infty}$-vector bundle on $X \backslash Y$ with the Hermitian metric given by the polarization.

2. Let us take a Riemannian metric $g$ on $X \backslash Y$ which behaves on a neighborhood of $Y$ as follows:

For any point $y_{0}$ of $Y$ let us take a local coordinate system $\left(z_{1}, \cdots, z_{n}\right)$ such that $Y$ is defined by $z_{1} \cdots z_{l}=0$. Then we assume

$$
g \sim \sum_{j \leq l} \frac{d z_{j} d \bar{z}_{j}}{\left(\left|z_{j}\right| \log \mid z_{j}\right)^{2}}+\sum_{j>l} d z_{j} d \bar{z}_{j} .
$$

Here $\sim$ means that each of the two metrics is bounded by a constant multiple of the other on a neighborhood of $y_{0}$. One can easily show (see [4]) that such a metric exists. If $X$ is a Kähler manifold, we can choose a Kähler metric as $g$.

3. Let us define the sheaf $\mathscr{D} b^{p}(H)$ on $X$ as follows:

For any open set $U$ of $X, \Gamma\left(U, \mathscr{D} b^{p}(H)\right)$ is the set of distribution-valued $p$-forms with coefficients in $H$ defined on $U \backslash Y$.

We also define the subsheaf $\mathcal{L}^{p}(H)_{(2)}$ of $\mathscr{D} b^{p}(H)$ as follows: 
For any open set $U$ of $X, \Gamma\left(U, \mathcal{L}^{p}(H)_{(2)}\right)$ is the set of $u \in \Gamma\left(U \backslash Y, \mathscr{D} b^{p}(H)\right)$ such that for any compact set $K$ of $U$, both $u$ and $d u$ are square-integrable on $K$. Here $d$ denotes the exterior derivative $\mathscr{D} b^{p}(H) \rightarrow \mathscr{D} b^{p+1}(H)$. The $L_{2}$ norm is defined, of course, by the Riemannian metric $g$ on $X$ and the Hermitian metric on the $C^{\infty}$-vector bundle $H$. Therefore if $X$ is compact, then

$\Gamma\left(X ; \mathcal{L}^{p}(H)_{(2)}\right)=\{u$; a distribution-valued $p$-form with coefficients in $H$ such that $u$ and $d u$ are square-integrable on $X \backslash Y\}$. It is clear that $\left\{\mathscr{D} b^{p}(H)\right\}_{p \in Z}$ forms a complex by the exterior derivative and $\left\{\mathcal{L}^{p}(H)_{(2)}\right\}$ forms its subcomplex.

4. Let us denote by ${ }^{\pi} H_{C}$, the minimal extension of $H_{C}=C \otimes_{Z} H_{Z}$. This means that ${ }^{\pi} H_{C}$ is a perverse complex on $X$ such that its restriction to $X \backslash Y$ coincides with $H_{C}$ and that there exists neither non-zero quotient nor subobject of ${ }^{\pi} H_{C}$ (in the category of perverse complexes) having its support in $Y$. Denoting by $\mathscr{D}_{X}$ the sheaf of linear differential operators on $X$, let us take a regular holonomic $\mathscr{D}_{X}$-module $\mathscr{M}$ such that its restriction to $X \backslash Y$ coincides with $\mathcal{O}_{X \backslash Y} \otimes H_{C}$ and that there exists neither non-zero coherent quotient nor submodule of $\mathscr{M}$ having its support in $Y$. Then we have

$$
{ }^{\pi} H_{C}=R \mathscr{H}_{\operatorname{om}_{\mathscr{D}_{X}}}\left(\mathcal{O}_{X}, \mathscr{M}\right) \text {. }
$$

Our main result is the following theorem.

Theorem 1. (i) $\mathcal{L}^{*}(H)_{(2)}$ is quasi-isomorphic to ${ }^{\pi} H$.

(ii) $H^{k}\left(X ; \mathcal{L}^{\cdot}(H)_{(2)}\right)=H^{k}\left(X ;{ }^{\pi} H\right)$.

Of course, (ii) is an immediate consequence of (i).

5. Outline of the proof of Theorem 1. The question being local, we consider the case where $X \backslash Y$ is the product of $n$ punctured discs. We may further assume that the monodromy of $H$ around $\left\{z_{j}=0\right\}$ is unipotent. We denote its logarithm by $N_{j}$. Now, by the coordinate transformation

$X \backslash Y$ is isomorphic to

$$
z_{j}=\exp \left(i x_{j}-t y_{j}\right)
$$

$$
\boldsymbol{Z}=\left\{(t, x, y) \in \boldsymbol{R}_{+} \times(\boldsymbol{R} / \boldsymbol{Z})^{n} \times \boldsymbol{R}_{+}^{n} ; 0<t<1, \sum y_{j}^{2}=1\right\}
$$

and the metric $g$ is equivalent to

$$
\frac{d t^{2}}{t^{2}}+t^{2}\left(\sum y_{j}^{2} d x_{j}^{2}\right)+\sum \frac{d y_{j}^{2}}{y_{j}^{2}}
$$

Let $V_{t_{0}}$ be the Riemannian manifold

equipped with the metric

$$
\left\{(t, x, y) \in Z ; t=t_{0}\right\}
$$

$$
t_{0}^{2}\left(\sum y_{j}^{2} d x_{j}^{2}\right)+\sum y_{j}^{-2} d y_{j}^{2}
$$

and denote $V_{1 / 2}$ by $V$. We trivialize the vector bundle $H$ so that we have

$$
d e=\sum N_{j} e \otimes d x_{j} \text {. }
$$

Let us choose $K \in \operatorname{End}(H)$ such that $\left[K, N_{j}\right]=-2 N_{j}$ and $\left.K\right|_{\mathrm{gr}_{k} W}=k$. Here $\operatorname{gr}_{k}^{W}$ denotes the graduation of the weight filtration $W=W\left(N_{1}, \cdots, N_{n}\right)$ determined by $N_{j}$ 's. Then we have ([2], [3])

Here $|*|_{Z}$ (resp., $|*|_{V}$ ) denotes the Hermitian metric on $\left.H\right|_{Z}$ (resp., $\left.H\right|_{V}$ ) at 
$(t, x, y)$ (resp., $(x, y))$.

To employ the harmonic analysis on $V_{t}$ we do some preliminary consideration on harmonic forms on $V_{t}$. Let $I_{k}$ denote the eigenspace of $K$ with eigenvalue $k$. We may, and do, suppose that the Hermitian metric on $H$ is chosen so that $\oplus_{k} I_{k}$ gives rise to an orthogonal decomposition of $H$.

Definition 1. For an $H$-valued form $u, A u$ is, by definition, $(K+2 p) u$, where $p$ denotes the degree of $u$ with respect to $d x_{1}, \cdots, d x_{n}$.

Then, for an $H$-valued form $u$,

$$
\|u\|_{Z}^{2}=\int\left|t^{-A / 2} u\right|^{2} d \mu_{Z}
$$

Here $d \mu_{Z}$ denotes the volume element of $Z$. We can now show the following

Lemma 1. Let $h$ be a harmonic form on $V_{t}$. Then $A h$ is also harmonic.

We now proceed by the induction on $n$. Then the $q$-th $L^{2}$-cohomology group $H_{(2)}^{q}(V ; H)$ coincides with the intersection cohomology group $H^{q}(V$; $\left.{ }^{\pi} H\right|_{V}$ ). In particular, we have the following

Lemma 2. $H^{q}\left(V ;\left.{ }^{\pi} H\right|_{V}\right)$ coincides with the space of $H$-valued $L^{2}-$ harmonic $q$-forms on $V$.

Furthermore we can prove the following

Lemma 3. The eigenvalue of $A$ in $H^{q}\left(V ;\left.{ }^{\pi} H\right|_{V}\right)$ is strictly greater than $n$ for $q \geqq n$ and strictly less than $n$ for $q<n$.

This lemma is a consequence of Theorem 2 in the next paragraph.

By Lemma 2 and Lemma 3 the proof of Theorem 1 is completed if we show that the $q$-th cohomology group of square integrable forms on $Z$ coincides with $H^{q}\left(V ;\left.{ }^{\pi} H\right|_{V}\right)$ for $q<n$ and vanishes for $q \geqq n$. To perform this we prove the following Lemma 4 by the harmonic analysis on $V_{t}$ and a subtle choice of partition of unity in the $t$-space.

Lemma 4. Let $\omega$ be a closed $L^{2}$-form on $Z$. Then we can find $h(t)$ and $h^{1}(t)$ which are harmonic forms on $V_{t}$ satisfying the following four conditions :

(4) $\omega-\left(h(t)+h^{1}(t) d t / t\right)$ is the coboundary of an $L^{2}$-form on $Z$,

(5) $\quad \partial h(t) / \partial t=0$,

(6) $h(t)$ is an $L^{2}$-form on $V_{t}$,

(7) $\quad h^{1}(t) d t / t$ is an $L^{2}$-form on $Z$.

By Hardy's inequality, we can find a square-integrable harmonic form $H^{1}$ such that $t \partial H^{1} / \partial t=h^{1}$. Furthermore the square-integrability condition implies $h=0$ for $q \geqq n$. Hence the $q$-th cohomology group of squareintegrable forms on $Z$ coincides with $H^{q}\left(V ;\left.{ }^{\pi} H\right|_{V}\right)$ for $q<n$ and vanishes for $q \geqq n$. This completes the proof of Theorem 1 .

6. Let $\operatorname{Im} N_{i_{1}} \cdots N_{i_{l}}$ be endowed with the quotient mixed Hodge structure of $H$ given by the weight filtration $W\left(N_{1}, \cdots, N_{n}\right)$. Let $\Pi\left(N_{1}, \cdots, N_{n}\right)$ be the following partial Koszul complex

$$
H \stackrel{N_{j}}{\longrightarrow} \oplus \operatorname{Im} N_{j} \stackrel{N_{k}}{\longrightarrow} \bigoplus_{j<k} \operatorname{Im} N_{j} N_{k} \longrightarrow \cdots \longrightarrow \operatorname{Im} N_{1} \cdots N_{n} .
$$


Then this is a complex in the category of mixed Hodge structures, and its $k$-th cohomology group $H^{k}\left(\Pi\left(N_{1}, \cdots, N_{n}\right)\right)$ coincides with the germ of $H^{k}\left({ }^{\pi} H\right)$ at the origin. Furthermore we have the following

Theorem 2. The weight of $H^{k}\left(\Pi\left(N_{1}, \cdots, N_{n}\right)\right)$ is equal to or less than $w+k$.

\section{References}

[1] Cattani, E. and A. Kaplan: Sur la cohomologie $L_{2}$ et la cohomologie d'intersection à coefficients dans une variation de structure de Hodge. C. R. Acad. Sci. Paris, 300, série I, 351-353 (1985).

[2] Cattani, E., A. Kaplan and W. Schmid: The $S L_{2}$-orbit theorem in several variables (to appear).

[3] Kashiwara, M.: The asymptotic behavior of a variation of Polarized Hodge structure (to appear in Publ. RIMS, Kyoto Univ.).

[4] Zucker, S.: Hodge theory with degenerating coefficients: $L_{2}$ cohomology in the Poincaré metric. Ann. of Math., 109, 415-476 (1979). 